Article

\title{
Synthesis, Characterization and Anti-Breast Cancer Activity of New 4-Aminoantipyrine-Based Heterocycles
}

\author{
Mostafa M. Ghorab ${ }^{1{ }^{*}}$, Marwa G. El-Gazzar ${ }^{2}$ and Mansour S. Alsaid ${ }^{1}$ \\ 1 Department of Pharmacognosy, College of Pharmacy, King Saud University, P.O. Box 2457, \\ Riyadh 11451, Saudi Arabia; E-Mail: msalsaid@ksu.edu.sa \\ 2 Department of Drug Radiation Research, National Center for Radiation Research and Technology, \\ Nasr City, Cairo 113701, Egypt; E-Mail: marwagalalgazzar@yahoo.com \\ * Author to whom correspondence should be addressed; E-Mail: mmsghorab@yahoo.com; \\ Tel.: +966-53-4292-860; Fax: +966-01-4670-560.
}

Received: 19 March 2014; in revised form: 9 April 2014 / Accepted: 17 April 2014 /

Published: 2 May 2014

\begin{abstract}
Aminoantipyrine was utilized as key intermediate for the synthesis of pyrazolone derivatives bearing biologically active moieties. The newly synthesized compounds were characterized by IR, ${ }^{1} \mathrm{H}$ - and ${ }^{13} \mathrm{C}$-NMR spectral and microanalytical studies. The compounds were screened as anticancer agents against a human tumor breast cancer cell line MCF7, and the results showed that (Z)-4-((3-amino-5-imino-1-phenyl-1H-pyrazol-4(5H)ylidene)methylamino)-1,5-dimethyl-2-phenyl-1,2-dihydropyrazol-3-one 5, 3-(4-bromophenyl) -1-(1,5-dimethyl-3-oxo-2-phenyl-2,3-dihydro-1H-pyrazol-4-yl)-4-oxo-2-thioxo-1,2,3,4tetrahydropyrimidine-5-carbonitrile 13, 1-(1,5-dimethyl-3-oxo-2-phenyl-2,3-dihydro- $1 H$ pyrazol-4-yl)-3-(4-iodophenyl)-4-oxo-2-thioxo-1,2,3,4-tetrahydropyrimidine-5-carbonitrile 14, 3,3'-(4,4'-sulfonylbis(4,1-phenylene))bis(1-(1,5-dimethyl-3-oxo-2-phenyl-2,3-dihydro- $1 H$ pyrazol-4-yl)-4-oxo-2-thioxo-1,2,3,4-tetrahydropyrimidine-5-carbonitrile) 16, (Z)-1(1,5-dimethyl-3-oxo-2-phenyl-2,3-dihydro-1H-pyrazol-4-yl)-2-hydrazono-4-oxo-3-phenyl1,2,3,4-tetrahydropyrimidine-5-carbonitrile $\quad \mathbf{1 7}$, (Z)-1-(1,5-dimethyl-3-oxo-2-phenyl2,3-dihydro-1 H-pyrazol-4-yl)-4-oxo-3-phenyl-2-(2-phenylhydrazono)-1,2,3,4-tetrahydro pyrimidine-5-carbonitrile 18, and (Z)-4-(3-amino-6-hydrazono-7-phenyl-6,7-dihydro pyrazolo[3,4- $d$ ]pyrimidin-5-yl)-1,5-dimethyl-2-phenyl-1,2-dihydropyrazol-3-one 19 were the most active compounds with $I C_{50}$ values ranging from 30.68 to $60.72 \mu \mathrm{M}$ compared with Doxorubicin as positive control with the $I C_{50}$ value $71.8 \mu \mathrm{M}$.
\end{abstract}


Keywords: pyrazoles; sulfonamides; anti-breast cancer

\section{Introduction}

Pyrazolone derivatives such as antipyrine, aminopyrine, and dipyrone are well known compounds used mainly as analgesic and antipyretic drugs and their pharmacological molecular mechanism has been widely surveyed [1,2]. One of the best known antipyrine derivatives is 4-aminoantipyrine which is used for the protection against oxidative stress as well as prophylactic of some diseases including cancer, and these are important directions in medical applications [3]. Several derivatives of antipyrine were also biologically evaluated, and analgesic [4], anti-inflammatory [5], antimicrobial [6], and anticancer activity [7-9] have been reported. Antipyrine derivatives are strong inhibitors of cycloxygenase isoenzymes, platelet tromboxane synthesis, and prostanoids synthesis [10], which catalyze the rate-limiting step of prostaglandin synthesis. Pyrazolones are also a well-known elicitor of hypersensitivity [11]. Recently, Al-Haiza et al. [12] synthesized a new compound with pyrazolone moiety with antimicrobial and antifungal activities. In the last decade, several pyrazole derivatives proved to have potent anticancer action by the inhibition of the cyclindependent kinases (CDKs) which are members of the large family of protein kinases and are responsible for the eukaryotic cell cycle regulation; they are intensively studied for their cancer implication [13]. Based on the above information and due to our interest in pyrazole as a biologically active pharmacophore [14-18], we synthesized a new series of heterocycles incorporating antipyrine moiety starting from 4-aminoantipyrine to be evaluated for their anticancer activity against human tumor breast cell line (MCF7).

\section{Results and Discussion}

\subsection{Chemistry}

The starting key reagent 4-aminoantipyrine was purchased from Sigma-Aldrich chemical company (St. Louis, MO, USA). In this work, the reactivity of 4-aminoantipyrine with active methylene containing compounds (malononitrile, 2-(ethoxymethylene)malononitrile, ethylcyanoacetate, (ethoxymethylene)ethylcyanoacetate and acetylacetone) was studied and the reaction proceeded in the presence of triethylorthoformate in methanol containing catalytic amounts of acetic acid following the reported reaction condition [19]. The obtained pyrazolone derivatives $\mathbf{2}-\mathbf{4}$, respectively, were identified by elemental and spectral data. Due to the biological importance of pyrazole, pyrrole and pyrimidine rings as anticancer agents [20-22], the pyrazolone derivative $\mathbf{2}$ was reacted with different nucleophiles in order to obtain biologically active pyrazole 5, pyrrole 6-8 and pyrimidine 9-16 derivatives bearing pyrazolone moieties. Thus, interaction of compound $\mathbf{2}$ with phenyl hydrazine yielded the corresponding pyrazole derivative 5. On the other hand, interaction of compound $\mathbf{2}$ with 2 -chloroacetonitrile, ethylbromoacetate or 2-chloroacetamide in dioxane containing a catalytic amount of triethylamine yielded the corresponding pyrrole derivatives 6-8, respectively. Reaction of compound $\mathbf{2}$ with thiourea in refluxing ethanol containing sodium ethoxide gave the corresponding pyrimidine derivative $\mathbf{9}$ (Scheme 1). The reactivity of compound $\mathbf{2}$ towards different aryl isothiocyanates/ $\mathrm{NaOH}$ was studied 
and the reaction proceeded via an addition reaction onto the isothiocyanato group followed by intramolecular cyclization to produce the pyrimidine ring as in compounds 10-15. Similarly, the bis-compound $\mathbf{1 6}$ was obtained expectedly via the reaction of one mole of 1-(4-isothio cyanatophenylsulfonyl)-4-isothiocyanatobenzene with two moles of compound $\mathbf{2}$ in ethanol containing sodium ethoxide (Scheme 2). The hydrazono pyrimidine derivatives 17, 18 were synthesized from compound 10 through reaction with either hydrazine hydrate or phenyl hydrazine, respectively, and the reaction proceeded via elimination of $\mathrm{H}_{2} \mathrm{~S}$ which was detected by a lead acetate paper. On the other hand, double cyclization took place when one mole of compound $\mathbf{1 0}$ was reacted with two moles of hydrazine hydrate to yield the corresponding pyrazolo[3,4-d]pyrimidine 19 (Scheme 3). Finally, treatment of 4-aminoantipyrine 1 with either triethylorthoformate or acetate in acetic anhydride resulted in the formation of compounds 20, 21, respectively (Scheme 4). The structures of compounds were proved by microanalytical and spectral data and were consistent with the proposed structures.

Scheme 1. Synthetic pathways for compounds 2-9.




Scheme 2. Synthetic pathways for compounds 10-16.

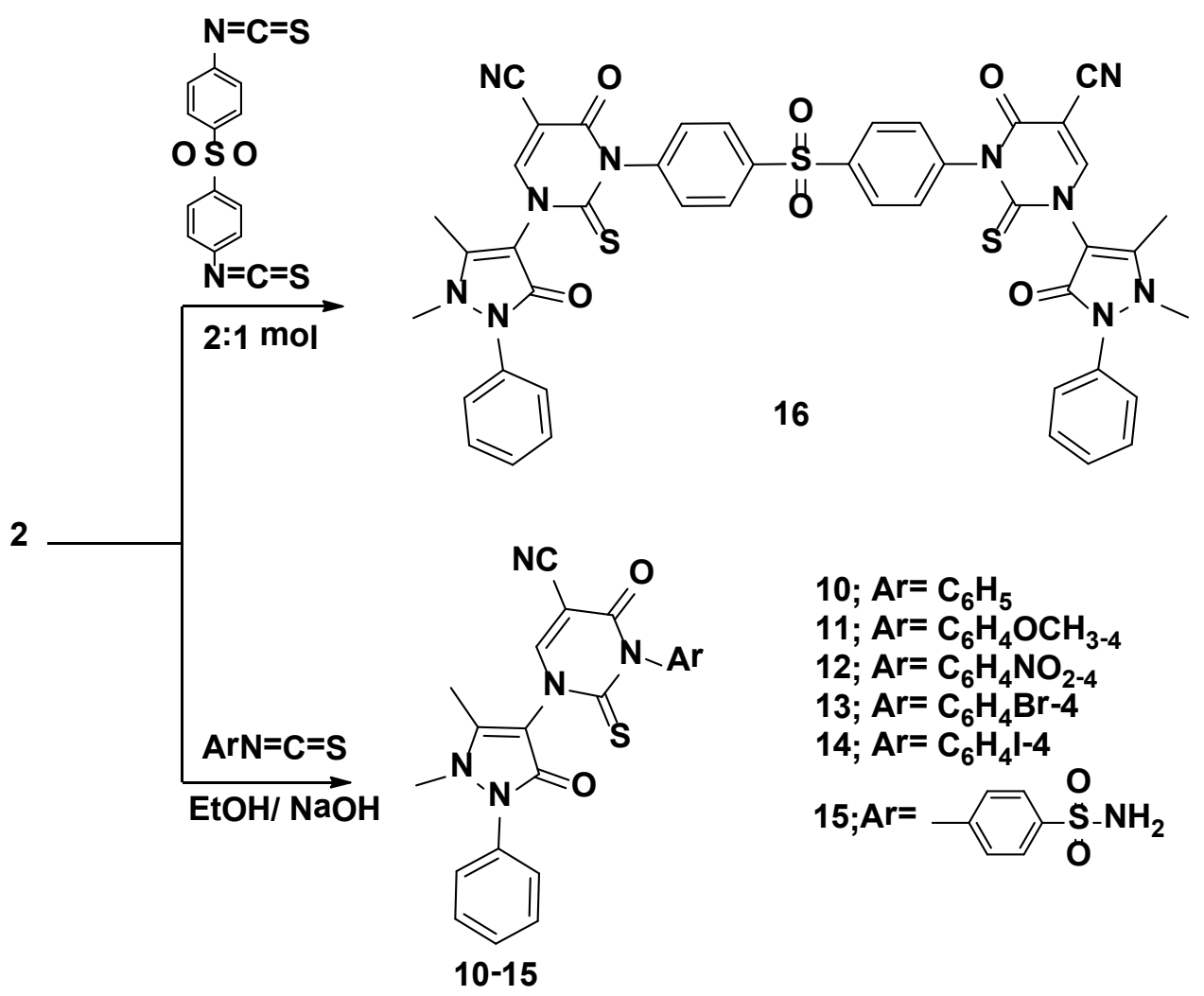

Scheme 3. Synthetic pathways for compounds 17-19.

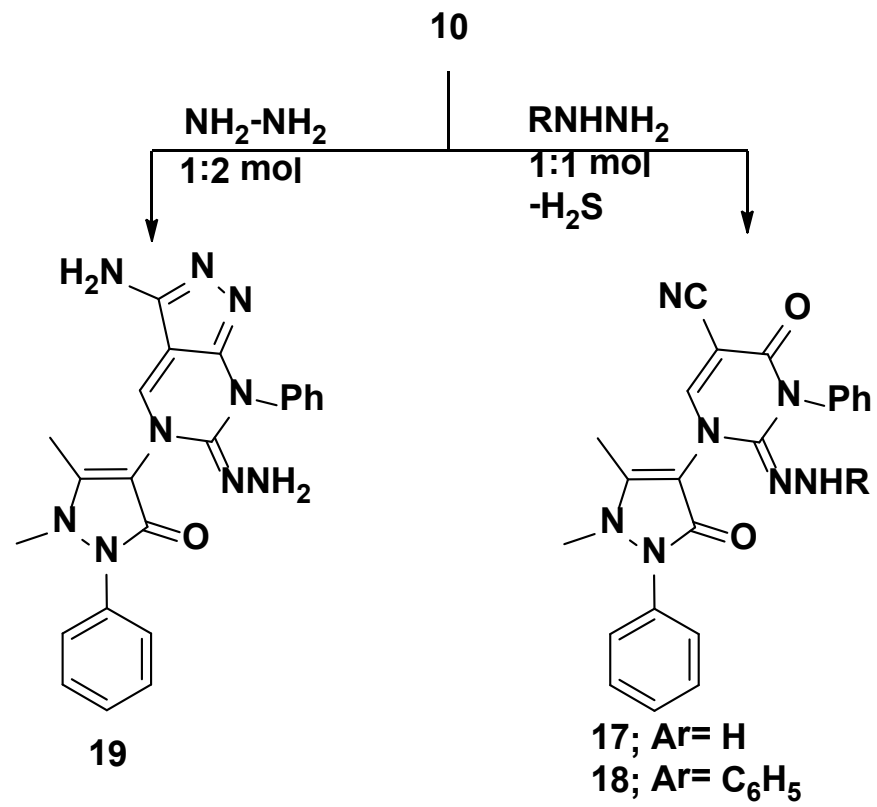


Scheme 4. Synthetic pathways for compounds 20, 21.

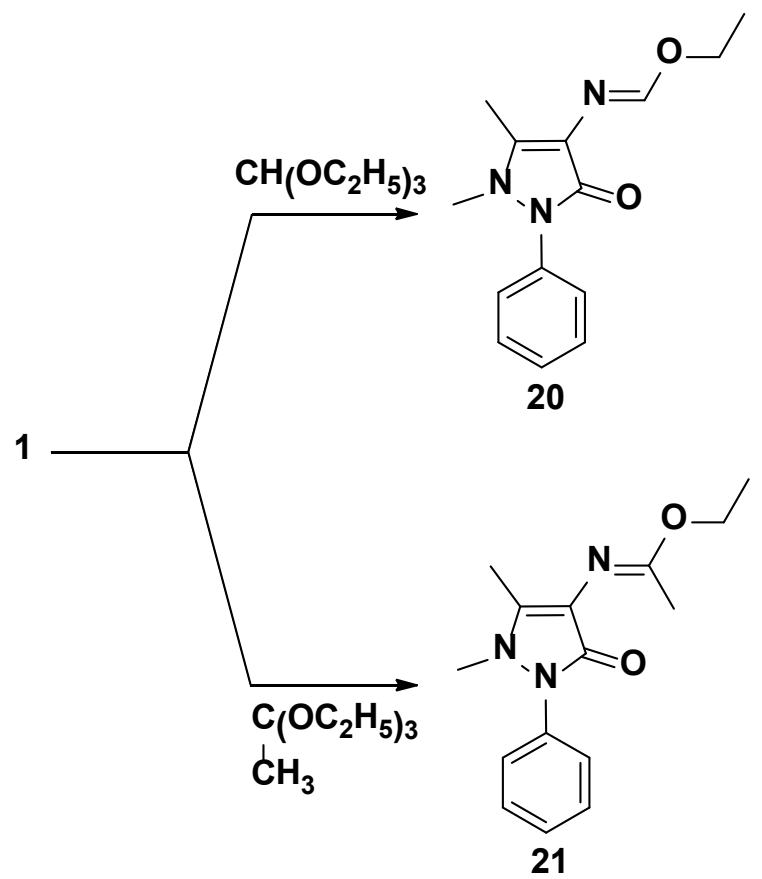

\subsection{In-Vitro Anticancer Screening}

Doxorubicin, the positive control used in this study, is an anticancer drug used to treat several cancer diseases including breast cancer. The relationship between surviving fraction and drug concentration was plotted; the response parameters calculated was $I C_{50}$ value, which corresponds to the compound concentration causing 50\% mortality in net cells (Table 1). The present work describes the synthesis of novel derivatives starting from 4-aminoantipyrine $\mathbf{1}$ by incorporating biologically active moieties, pyrazole, pyrrole, pyrimidine and pyrazolopyrimidine. From Table 1, we can observe that some of the tested compounds were found to be equipotent or even more potent than Doxorubicin on MCF7 cell line with $I C_{50}$ values ranging from 30.68 to $70.65 \mu \mathrm{M}$ compared to the reference drug $(71.8 \mu \mathrm{M})$. The most potent compounds in this study were found to be those belonging to the pyrimidine derivatives of antipyrine especially the halogenated ones, where, the iodophenyl 14 $\left(I C_{50}=30.68 \mu \mathrm{M}\right)$ and the bromophenyl $13\left(I C_{50}=43.41 \mu \mathrm{M}\right)$ derivatives showed significant activities, also, the biscompound $\mathbf{1 6}\left(I C_{50}=37.22 \mu \mathrm{M}\right)$. In addition, pyrazole 5, pyrimidines 17, 18, pyrazolopyrimidine 19 with $I C_{50}$ values $60.72,54.23,44.99$, and $44.49 \mu \mathrm{M}$ exhibited a higher activity when compared with the Doxorubicin as positive control. The phenyl and 4-nitro phenyl derivatives 10 and $12\left(I C_{50}=72.04\right.$ and $\left.70.65 \mu \mathrm{M}\right)$ are nearly as active as Doxorubicin. On the other hand, reaction of one mole of hydrazine hydrate or phenyl hydrazine with compound $\mathbf{1 0}$ yielded the pyrimidine derivatives 17 and 18 with significant activities $\left(I C_{50}=54.23\right.$ and $\left.44.99 \mu \mathrm{M}\right)$, while, addition of two moles of hydrazine hydrate yielded the pyrazolopyrimidine $19\left(I C_{50}=44.49 \mu \mathrm{M}\right)$ which is nearly as potent as compounds $\mathbf{1 7}$ and $\mathbf{1 8}$. Moreover, the reaction of phenylhydrazine with compound $\mathbf{2}$ was also successful as it yielded the pyrazole derivative 5 which showed high activity $\left(I C_{50}=60.72 \mu \mathrm{M}\right)$. All these compounds are more active than the reference drug. Considering the pyrrole derivatives 6, 7 and $\mathbf{8}$, they were found to exhibit lower activity compared to the reference drug $\left(I C_{50}=128.61,104.11\right.$ 
and $85.12 \mu \mathrm{M}$, respectively). Compounds $\mathbf{2 - 4}, \mathbf{9}, \mathbf{1 1}, \mathbf{1 5}, \mathbf{2 0}$ and 21 are the least potent in this study with $I C_{50}$ ranging from 109.58 to $173.83 \mu \mathrm{M}$.

Table 1. In-vitro anticancer screening of compounds 2-21 against human breast cell line (MCF7).

\begin{tabular}{ccc}
\hline Compound No. & $\boldsymbol{I C}_{\mathbf{5 0}}(\boldsymbol{\mu g} / \mathbf{m L})$ & $\boldsymbol{I C}_{\mathbf{5 0}}(\boldsymbol{\mu M})$ \\
\hline $\mathbf{2}$ & 48.2 & 173.83 \\
$\mathbf{3}$ & 38.0 & 116.56 \\
$\mathbf{4}$ & 34.9 & 111.50 \\
$\mathbf{5}$ & 23.5 & 60.72 \\
$\mathbf{6}$ & 40.9 & 128.61 \\
$\mathbf{7}$ & 38.0 & 104.11 \\
$\mathbf{8}$ & 28.6 & 85.12 \\
$\mathbf{9}$ & 38.9 & 109.58 \\
$\mathbf{1 0}$ & 29.9 & 72.04 \\
$\mathbf{1 1}$ & $\mathrm{NA}$ & $\mathrm{NA}$ \\
$\mathbf{1 2}$ & 32.5 & 70.65 \\
$\mathbf{1 3}$ & 21.4 & 43.41 \\
$\mathbf{1 4}$ & 16.6 & 30.68 \\
$\mathbf{1 5}$ & $\mathrm{NA}$ & $\mathrm{NA}$ \\
$\mathbf{1 6}$ & 33.2 & 37.22 \\
$\mathbf{1 7}$ & 22.4 & 54.23 \\
$\mathbf{1 8}$ & 22.0 & 44.99 \\
$\mathbf{1 9}$ & 19.0 & 44.49 \\
$\mathbf{2 0}$ & 35.8 & 138.22 \\
$\mathbf{2 1}$ & $\mathrm{NA}$ & $\mathrm{NA}$ \\
\hline Doxorubicin & 39.0 & 71.8 \\
\hline NA: Compound having $I C_{50}$ value $>100 \mu \mathrm{g} / \mathrm{mL}$. \\
\hline
\end{tabular}

Generally, incorporation of pyrimidine ring yielded the most potent compounds and these results point to the possible use of pyrimidine derivatives of antipyrine for treatment of breast tumors.

\section{Experimental Section}

\subsection{General}

Reagents were obtained from commercial suppliers and were used without purification. Melting points were determined in open capillary tubes using Thermo system FP800 Mettler FP80 central processor supplied with FP81 MBC cell apparatus (Stuart Scientific, Redhill, UK), and were uncorrected. Elemental analyses $(\mathrm{C}, \mathrm{H}, \mathrm{N})$ were performed on a Perkin-Elmer 2400 Instrument (Perkin-Elmer, Norwalk, CT, USA). All compounds were within $\pm 0.4 \%$ of the theoretical values. Infrared (IR) spectra (KBr disc) were recorded on FT-IR spectrophotometer (Perkin Elmer, Norwalk, CT, USA) at the Research Center, College of Pharmacy, King Saud University, Saudi Arabia. ${ }^{1} \mathrm{H}$ and ${ }^{13} \mathrm{C}$ NMR spectra were recorded on a Ultra Shield Plus $500 \mathrm{MHz}$ (Bruker, Munich, Germany) spectrometer operating at $500 \mathrm{MHz}$ for proton and $125 \mathrm{MHz}$ for carbon, respectively. The chemical shift values are reported in $\delta$ (ppm) relative to the residual solvent peak, the coupling constants $(J)$ are reported in Hertz $(\mathrm{Hz})$. 


\subsection{Chemistry}

3.2.1. 2-((1,5-Dimethyl-3-oxo-2-phenyl-2,3-dihydro-1H-pyrazol-4-ylamino)methylene)-malononitrile 2

A mixture of 4-aminoantipyrine $(0.01 \mathrm{~mol})$, malononitrile $(0.01 \mathrm{~mol})$, triethylorthoformate $(0.01 \mathrm{~mol})$, and acetic acid $(1 \mathrm{~mL})$ in methanol $(30 \mathrm{~mL})$ was refluxed for $5 \mathrm{~h}$, the reaction mixture was cooled, filtered, the filtered solid was crystallized from ethanol to give 2 . Yield\%: 90, m.p. $=254.7^{\circ} \mathrm{C}$, IR, $\mathrm{cm}^{-1}: 3210$ (NH), 3055 (CH arom.), 2941, 2818 (CH aliph.), $2200(\mathrm{CN}), 1658$ (C=N). ${ }^{1} \mathrm{H}$ (DMSO- $d_{6}$, ppm): $2.2\left[\mathrm{~s}, 3 \mathrm{H}, \mathrm{CH}_{3}\right], 3.2\left[\mathrm{~s}, 3 \mathrm{H}, \mathrm{N}-\mathrm{CH}_{3}\right], 7.3-7.9[\mathrm{~m}, 5 \mathrm{H}, \mathrm{Ar}-\mathrm{H}], 8.1[\mathrm{~s}, 1 \mathrm{H}, \mathrm{CH}], 10.5[\mathrm{~s}, 1 \mathrm{H}, \mathrm{NH}$, $\mathrm{D}_{2} \mathrm{O}$-exchangeable]. ${ }^{13} \mathrm{C}$ (DMSO- $\left.d_{6}, \mathrm{ppm}\right): 10.3\left(\mathrm{CH}_{3}\right), 29.1\left(\mathrm{~N}-\mathrm{CH}_{3}\right), 50.3,104.6,114.1(2), 116.4(2)$, 117.2, 127.2(2), 129.1, 134.5, $160.1(\mathrm{C}=\mathrm{O})$, 165.5. Anal. Calcd. for $\mathrm{C}_{15} \mathrm{H}_{13} \mathrm{~N}_{5} \mathrm{O}$ (279): $\mathrm{C}, 64.51$; H, 4.69; N, 25.07. Found: C, 64.91; H, 4.80; N, 25.34.

\subsection{2. (Z)-Ethyl-2-cyano-3-(1,5-dimethyl-3-oxo-2-phenyl-2,3-dihydro-1H-pyrazol-4-ylamino)acrylate 3}

A mixture of 4-aminoantipyrine $(0.01 \mathrm{~mol})$, ethylcyanoacetate $(0.01 \mathrm{~mol})$, triethylorthoformate $(0.01 \mathrm{~mol})$, and acetic acid $(1 \mathrm{~mL})$ in methanol $(30 \mathrm{~mL})$ was refluxed for $5 \mathrm{~h}$, the reaction mixture was filtered, the filtered solid was crystallized from ethanol to give 3. Yield\%: 83, m.p. $=154.8{ }^{\circ} \mathrm{C}$, IR, $\mathrm{cm}^{-1}$ : 3192 (NH), 3065 (CH arom.), 2965, 2841 (CH aliph.), $2214(\mathrm{CN}), 1710,1658(2 \mathrm{C}=\mathrm{O})$. ${ }^{1} \mathrm{H}\left(\mathrm{DMSO}-d_{6}, \mathrm{ppm}\right): 1.2\left[\mathrm{t}, 3 \mathrm{H}, \mathrm{CH}_{3}, J=7.9 \mathrm{~Hz}\right], 2.3\left[\mathrm{~s}, 3 \mathrm{H}, \mathrm{CH}_{3}\right], 3.2\left[\mathrm{~s}, 3 \mathrm{H}, \mathrm{N}-\mathrm{CH}_{3}\right], 4.2[\mathrm{q}, 2 \mathrm{H}$, $\left.\mathrm{CH}_{2}, J=8.1 \mathrm{~Hz}\right], 7.3-8.0[\mathrm{~m}, 5 \mathrm{H}, \mathrm{Ar}-\mathrm{H}], 8.2[\mathrm{~d}, 1 \mathrm{H}, \mathrm{CH}, J=7.12 \mathrm{~Hz}], 10.0[\mathrm{~d}, 1 \mathrm{H}, \mathrm{NH}$, $\mathrm{D}_{2} \mathrm{O}$-exchangeable, $\left.J=7.3 \mathrm{~Hz}\right] .{ }^{13} \mathrm{C}$ (DMSO- $\left.d_{6}, \mathrm{ppm}\right): 10.3,14.3,39.0,60.2,72.6,109.7,115.7(2)$, 116.0, 118,1, 129.0(2), 129.1, 134.3, 156.8, 161.0, 166.3. Anal. Calcd. for $\mathrm{C}_{17} \mathrm{H}_{18} \mathrm{~N}_{4} \mathrm{O}_{3}(326)$ : C, 62.57; H, 5.56; N, 17.17. Found: C, 62.90; H, 5.89; N, 17.25 .

3.2.3. 3-((1,5-Dimethyl-3-oxo-2-phenyl-2,3-dihydro-1H-pyrazol-4-ylamino)methylene)-pentane2,4-dione 4

A mixture of 4-aminoantipyrine $(0.01 \mathrm{~mol})$, acetylacetone $(0.01 \mathrm{~mol})$, triethylorthoformate $(0.01 \mathrm{~mol})$, and acetic acid $(1 \mathrm{~mL})$ in methanol $(30 \mathrm{~mL})$ was refluxed for $5 \mathrm{~h}$, the reaction mixture was filtered, the filtered solid was crystallized from ethanol to give 4. Yield\%: 90, m.p. $=201.8{ }^{\circ} \mathrm{C}$, IR, $\mathrm{cm}^{-1}$ : 3209 (NH), 3100 (CH arom.), 2971, 2848 (CH aliph.), 1716, 1692, 1661 (3C=O), $1612(\mathrm{C}=\mathrm{N}) .{ }^{1} \mathrm{H}\left(\mathrm{DMSO}-d_{6}, \mathrm{ppm}\right): 1.9\left[\mathrm{~s}, 3 \mathrm{H}, \mathrm{CH}_{3}\right], 2.1\left[\mathrm{~s}, 6 \mathrm{H}, 2 \mathrm{COCH}_{3}\right], 3.0\left[\mathrm{~s}, 3 \mathrm{H}, \mathrm{N}-\mathrm{CH}_{3}\right]$, 7.3-7.5 [m, 6H, Ar-H $+\mathrm{CH}], 9.0\left[\mathrm{~s}, 1 \mathrm{H}, \mathrm{NH}, \mathrm{D}_{2} \mathrm{O}-\right.$ exchangeable $] .{ }^{13} \mathrm{C}$ (DMSO- $\left.d_{6}, \mathrm{ppm}\right): 11.1$, 22.4(2), 39.1, 104.4, 107.8(2), 108.4, 121.8, 128.8(2), 129.0, 135.0, 151.0, 161.8, 195.1(2). Anal. Calcd. for $\mathrm{C}_{17} \mathrm{H}_{19} \mathrm{~N}_{3} \mathrm{O}_{3}$ (313): C, 65.16; H, 6.11; N, 13.41. Found: C, 65.87; H, 6.37; N, 13.21 .

3.2.4. (Z)-4-((3-Amino-5-imino-1-phenyl-1H-pyrazol-4(5H)-ylidene)methylamino)-1,5-dimethyl-2phenyl-1,2-dihydropyrazol-3-one 5

Compound $2(0.01 \mathrm{~mol})$ was mixed with phenyl hydrazine $(0.01 \mathrm{~mol})$ in dioxane $(20 \mathrm{~mL})$ and refluxed for $5 \mathrm{~h}$, the reaction mixture was cooled, poured onto ice water. The precipitated solid products were filtered and crystallized from methanol to give compound 5. Yield\%: 76, m.p. $=172.7{ }^{\circ} \mathrm{C}$, IR, $\mathrm{cm}^{-1}$ : 3386, 3348, $3276\left(\mathrm{NH}, \mathrm{NH}_{2}\right), 3026$ (CH arom.), 2981, 2872 ( $\mathrm{CH}$ aliph.), $1660(\mathrm{C}=\mathrm{O})$, 
$1595(\mathrm{C}=\mathrm{N}) .{ }^{1} \mathrm{H}\left(\mathrm{DMSO}-d_{6}, \mathrm{ppm}\right): 2.2\left[\mathrm{~s}, 3 \mathrm{H}, \mathrm{CH}_{3}\right], 3.2\left[\mathrm{~s}, 3 \mathrm{H}, \mathrm{N}-\mathrm{CH}_{3}\right], 7.2-7.9$ [m, 10H, $\left.\mathrm{Ar}-\mathrm{H}\right]$, $8.3[\mathrm{~s}, 1 \mathrm{H}, \mathrm{CH}], 8.7$ [s, 1H, NH, $\mathrm{D}_{2} \mathrm{O}$-exchangeable], 13.2 [s, 1H, NH-imino, $\mathrm{D}_{2} \mathrm{O}$-exchangeable]. ${ }^{13} \mathrm{C}$ (DMSO-d $\left.\mathrm{d}_{6}, \mathrm{ppm}\right): 11.5,39.5,106.2,108.0,112.4(2), 116.1(2), 117.6,124.1,127.7(2), 128.9$, 129.5(2), 137.9, 141.2, 148.4, 156.5, 163.1, 166.2. Anal. Calcd. for $\mathrm{C}_{21} \mathrm{H}_{21} \mathrm{~N}_{7}$ (387): C, 65.10; $\mathrm{H}, 5.46$; N, 25.31. Found: C, 65.32; H, 5.09; N, 25.55.

\subsubsection{3-Amino-1-(1.5-dimethyl-3-oxo-2-phenyl-2,3-dihydro-1 $H$-pyrazol-4-yl)-1H-pyrrole-2,4-} dicarbonitrile 6

Compound $2(0.01 \mathrm{~mol})$ was mixed with 2-chloroacetonitrile $(0.01 \mathrm{~mol})$ in dioxane $(20 \mathrm{~mL})$ containing 3 drops of triethylamine and refluxed for $5 \mathrm{~h}$, the reaction mixture was cooled, poured onto ice water. The precipitated solid products were filtered and crystallized from methanol to give compound 6. Yield\%: 70, m.p. $>350{ }^{\circ} \mathrm{C}$, IR, $\mathrm{cm}^{-1}: 3344,3291\left(\mathrm{NH}_{2}\right), 3100$ (CH arom.), 2966, 2881 (CH aliph.), $2200(\mathrm{CN}), 1699(\mathrm{C}=\mathrm{O}) .{ }^{1} \mathrm{H}\left(\mathrm{DMSO}-d_{6}, \mathrm{ppm}\right): 1.6\left[\mathrm{~s}, 3 \mathrm{H}, \mathrm{CH}_{3}\right], 2.4$ [s, 3H, N-CH $\mathrm{CH}_{3}$, 6.5 [s, 2H, $\mathrm{NH}_{2}, \mathrm{D}_{2} \mathrm{O}$-exchangeable], 7.0-8.0 [m, 6H, Ar-H + CH-pyrrole $] .{ }^{13} \mathrm{C}$ (DMSO-d $\left.d_{6}, \mathrm{ppm}\right)$ : 13.9, 39.1, 101.2(2), 107.4, 110.4, 113.9(2), 116.2, 117.6(2), 119.1, 129.3(2), 138.1, 139.4, 161.2. Anal. Calcd. for $\mathrm{C}_{17} \mathrm{H}_{14} \mathrm{~N}_{6} \mathrm{O}$ (318): C, 64.14; H, 4.43; N, 25.40. Found: C, 64.32; H, 4.67; N, 25.12.

3.2.6. Ethyl-3-amino-4-cyano-1-(1,5-dimethyl-3-oxo-2-phenyl-2,3-dihydro-1 $H$-pyrazol-4-yl)- $1 H$ pyrrole-2-carboxylate 7

Compound 2 (0.01 mol) was mixed with ethyl bromoacetate $(0.01 \mathrm{~mol})$ in dioxane $(20 \mathrm{~mL})$ containing 3 drops of triethylamine and refluxed for $5 \mathrm{~h}$, the reaction mixture was cooled, poured onto ice water. The precipitated solid products were filtered and crystallized from methanol to give compound 7. Yield\%: 89, m.p. $=143.4{ }^{\circ} \mathrm{C}, \mathrm{IR}, \mathrm{cm}^{-1}$ : $3411,3396\left(\mathrm{NH}_{2}\right), 3100(\mathrm{CH}$ arom. $), 2976$, 2882 (CH aliph.), $2186(\mathrm{CN}), 1718,1696(2 \mathrm{C}=\mathrm{O}) .{ }^{1} \mathrm{H}\left(\mathrm{DMSO}-d_{6}, \mathrm{ppm}\right): 1.2\left[\mathrm{t}, 3 \mathrm{H}, \mathrm{CH}_{3}-\mathrm{ester}\right]$, $2.0\left[\mathrm{~s}, 3 \mathrm{H}, \mathrm{CH}_{3}\right], 2.4\left[\mathrm{~s}, 3 \mathrm{H}, \mathrm{N}-\mathrm{CH}_{3}\right], 4.1$ [q, 2H, $\left.\mathrm{CH}_{2}-\mathrm{ester}\right], 7.3-8.0$ [m, 7H, $\mathrm{Ar}-\mathrm{H}+\mathrm{NH}_{2}$ ], 8.7 $\left[\mathrm{s}, 1 \mathrm{H}, \mathrm{CH}-\right.$ pyrrole]. ${ }^{13} \mathrm{C}$ (DMSO- $\left.d_{6}, \mathrm{ppm}\right): 12.6,13.4,39.0,59.8,98.7,112.0,114.3(2), 115.6,118.4$, 122.7, 126.3, 129.1(2), 138.1, 139.6, 141.2, 160.0, 161.2. Anal. Calcd. for $\mathrm{C}_{19} \mathrm{H}_{19} \mathrm{~N}_{5} \mathrm{O}_{3}(365)$ : C, 62.46; H, 5.24; N, 19.17. Found: C, 62.79; H, 5.57; N, 19.34 .

3.2.7. 3-Amino-4-cyano-1-(1,5-dimethyl-3-oxo-2-phenyl-2,3-dihydro-1H-pyrazol-4-yl)- $1 H$-pyrrole-2carboxamide 8

Compound 2 (0.01 mol) was mixed with 2-chloroacetamide $(0.01 \mathrm{~mol})$ in dioxane $(20 \mathrm{~mL})$ containing 3 drops of triethylamine and refluxed for $5 \mathrm{~h}$, the reaction mixture was cooled, poured onto ice water. The precipitated solid products were filtered and crystallized from methanol to give compound 8. Yield\%: 76, m.p. > $350{ }^{\circ} \mathrm{C}, \mathrm{IR}, \mathrm{cm}^{-1}$ : 3404, 3385, $3236\left(\mathrm{NH}_{2}\right), 3066$ (CH arom.), 2961, 2836 (CH aliph.), $2196(\mathrm{CN}), 1700,1680(2 \mathrm{C}=\mathrm{O}) .{ }^{1} \mathrm{H}\left(\mathrm{DMSO}-d_{6}, \mathrm{ppm}\right): 1.6\left[\mathrm{~s}, 3 \mathrm{H}, \mathrm{CH}_{3}\right], 2.3[\mathrm{~s}, 3 \mathrm{H}$, $\left.\mathrm{N}-\mathrm{CH}_{3}\right], 7.2-8.0\left[\mathrm{~m}, 9 \mathrm{H}, \mathrm{Ar}-\mathrm{H}+\mathrm{CONH}_{2}+\mathrm{NH}_{2}\right], 8.5$ [s, $1 \mathrm{H}, \mathrm{CH}-$ pyrrole]. ${ }^{13} \mathrm{C}$ (DMSO- $\left.d_{6}, \mathrm{ppm}\right)$ : 11.9, 39.1, 99.2, 111.8, 114.3(2), 115.6, 118.2, 124.4, 129.0(2), 131.8, 135.7, 138.2, 153.2, 162.4, 166.0. Anal. Calcd. for $\mathrm{C}_{17} \mathrm{H}_{16} \mathrm{~N}_{6} \mathrm{O}_{3}$ (336): C, 60.71; H, 4.79; N, 24.99. Found: C, 60.99; H, 4.49; N, 24.70. 
3.2.8. 4-((4,6-Diamino-2-thioxopyrimidin-5(2H)-ylidene)methylamino)-1,5-dimethyl-1,2-phenyl-1,2dihydropyrazol-3-one 9

A mixture of $2(0.01 \mathrm{~mol})$ and thiourea $(0.01 \mathrm{~mol})$ was refluxed for $5 \mathrm{~h}$ in ethanol containing sodium ethoxide $(0.01 \mathrm{~mol})$. The reaction mixture was cooled, poured onto ice water, acidified with dil. $\mathrm{HCl}$, the precipitated solid product was filtered and crystallized from methanol to give 9. Yield\%: 90, m.p. $=219.6{ }^{\circ} \mathrm{C}, \mathrm{IR}, \mathrm{cm}^{-1}: 3410,3362,3350\left(\mathrm{NH}_{2}\right), 3054$ (CH arom.), 2922, 2860 (CH aliph.), $1675(\mathrm{C}=\mathrm{O}), 1624(\mathrm{C}=\mathrm{N}), 1317(\mathrm{C}=\mathrm{S}) .{ }^{1} \mathrm{H}\left(\mathrm{DMSO}-d_{6}, \mathrm{ppm}\right): 2.2\left[\mathrm{~s}, 3 \mathrm{H}, \mathrm{CH}_{3}\right], 3.1$ [s, 3H, N-CH$]$, $7.3\left[\mathrm{~s}, 4 \mathrm{H}, 2 \mathrm{NH}_{2}, \mathrm{D}_{2} \mathrm{O}\right.$-exchangeable], 7.5-7.9 [m, 5H, Ar-H], $9.4[\mathrm{~s}, 1 \mathrm{H}, \mathrm{CH}], 13.0[\mathrm{~s}, 1 \mathrm{H}, \mathrm{NH}$, $\mathrm{D}_{2} \mathrm{O}$-exchangeable]. ${ }^{13} \mathrm{C}$ (DMSO- $\left.d_{6}, \mathrm{ppm}\right): 10.3,39.3,84.2,109.1,116.4(2), 124.2,127.2(2), 129.1$, 134.4(2), 160.2, 170.8(2), $211.3(\mathrm{C}=\mathrm{S})$. Anal. Calcd. for $\mathrm{C}_{16} \mathrm{H}_{17} \mathrm{~N}_{7} \mathrm{OS}$ (355): C, 54.07; H, 4.82; N, 27.59. Found: C, 54.34; H, 4.65; N, 27.25.

\subsection{General Procedure for Preparation of Compounds 10-15}

A mixture of $2(0.01 \mathrm{~mol})$ and the appropriate aryl isothiocyanate $(0.01 \mathrm{~mol})$ was refluxed for $10 \mathrm{~h}$ in ethanol containing sodium ethoxide $(0.01 \mathrm{~mol})$. The reaction mixture was cooled, poured onto ice water, acidified with dil. $\mathrm{HCl}$, the precipitated solid product was filtered and crystallized from methanol to give 10-15, respectively.

3.3.1. 1-(1,5-Dimethyl-3-oxo-2-phenyl-2,3-dihydro-1H-pyrazol-4-yl)-4-oxo-3-phenyl-2-thioxo1,2,3,4-tetrahydropyrimidine-5-carbonitrile $\mathbf{1 0}$

Yield\%: 81, m.p. $=132.9{ }^{\circ} \mathrm{C}, \mathrm{IR}, \mathrm{cm}^{-1}$ : $3100(\mathrm{CH}$ arom.), 2966, $2871(\mathrm{CH}$ aliph.), $2201(\mathrm{CN})$, 1672, $1659(2 \mathrm{C}=\mathrm{O}), 1292(\mathrm{C}=\mathrm{S}) .{ }^{1} \mathrm{H}\left(\mathrm{DMSO}-d_{6}, \mathrm{ppm}\right): 2.2\left[\mathrm{~s}, 3 \mathrm{H}, \mathrm{CH}_{3}\right], 3.2\left[\mathrm{~s}, 3 \mathrm{H}, \mathrm{N}-\mathrm{CH}_{3}\right], 7.3$ [s, $1 \mathrm{H}, \mathrm{CH}-$ pyrimidine $], 7.5-8.1[\mathrm{~m}, 10 \mathrm{H}, \mathrm{Ar}-\mathrm{H}] .{ }^{13} \mathrm{C}$ (DMSO- $\left.d_{6}, \mathrm{ppm}\right): 14.4,39.1,104.6,109.3$, 114.1(2), 116.5, 118.1, 122.0(2), 126.3, 128.6(2), 129.1(2), 129.3, 134.5, 137.8, 139.1, 159.9, 160.5, $187.2(\mathrm{C}=\mathrm{S})$. Anal. Calcd. for $\mathrm{C}_{22} \mathrm{H}_{17} \mathrm{~N}_{5} \mathrm{O}_{2} \mathrm{~S}$ (415): C, 63.60; H, 4.12; N, 16.86. Found: C, 63.34; $\mathrm{H}, 4.32 ; \mathrm{N}, 16.60$.

3.3.2. 1-(1,5-Dimethyl-3-oxo-2-phenyl-2,3-dihydro-1H-pyrazol-4-yl)-3-(4-methoxyphenyl)-4-oxo-2thioxo-1,2,3,4-tetrahydropyrimidine-5-carbonitrile 11

Yield\%: 71, m.p. $=90.6{ }^{\circ} \mathrm{C}, \mathrm{IR}, \mathrm{cm}^{-1}: 3046(\mathrm{CH}$ arom.), 2991, $2875(\mathrm{CH}$ aliph.), $2208(\mathrm{CN}), 1684$, $1654(2 \mathrm{C}=\mathrm{O}), 1257(\mathrm{C}=\mathrm{S}) .{ }^{1} \mathrm{H}\left(\mathrm{DMSO}-d_{6}, \mathrm{ppm}\right): 2.4\left[\mathrm{~s}, 3 \mathrm{H}, \mathrm{CH}_{3}\right], 3.3\left[\mathrm{~s}, 3 \mathrm{H}, \mathrm{N}-\mathrm{CH}_{3}\right], 3.7[\mathrm{~s}, 3 \mathrm{H}$, $\left.\mathrm{OCH}_{3}\right], 6.8-7.5[\mathrm{~m}, 10 \mathrm{H}, \mathrm{Ar}-\mathrm{H}+\mathrm{CH}-$ pyrimidine $] .{ }^{13} \mathrm{C}$ (DMSO- $\left.d_{6}, \mathrm{ppm}\right): 13.9,39.1,55.1,95.2,107.5$, 113.5(2), 113.8(3), 123.3, 124.2(2), 124.9, 129.1(2), 130.8, 131.4, 143.6, 156.2, 156.6, 164.7, 188.0. Anal. Calcd. for $\mathrm{C}_{23} \mathrm{H}_{19} \mathrm{~N}_{5} \mathrm{O}_{3} \mathrm{~S}$ (445): C, 62.01; H, 4.30; N, 15.72. Found: C, 62.34; H, 4.09; N, 15.51.

3.3.3. 1-(1,5-Dimethyl-3-oxo-2-phenyl-2,3-dihydro-1H-pyrazol-4-yl)-3-(4-nitrophenyl)-4-oxo-2thioxo-1,2,3,4-tetrahydropyrimidine-5-carbonitrile $\mathbf{1 2}$

Yield\%: 79, m.p. $=190.9{ }^{\circ} \mathrm{C}, \mathrm{IR}, \mathrm{cm}^{-1}$ : $3076(\mathrm{CH}$ arom.), 2991, $2908(\mathrm{CH}$ aliph.), $2188(\mathrm{CN})$, 1700, $1689(2 \mathrm{C}=\mathrm{O}), 1597,1380\left(\mathrm{NO}_{2}\right), 1308(\mathrm{C}=\mathrm{S}) .{ }^{1} \mathrm{H}\left(\mathrm{DMSO}-d_{6}, \mathrm{ppm}\right): 2.5\left[\mathrm{~s}, 3 \mathrm{H}, \mathrm{CH}_{3}\right], 3.3[\mathrm{~s}, 3 \mathrm{H}$, 
$\left.\mathrm{N}-\mathrm{CH}_{3}\right], 7.8-8.2[\mathrm{~m}, 10 \mathrm{H}, \mathrm{Ar}-\mathrm{H}+\mathrm{CH}-$ pyrimidine $] .{ }^{13} \mathrm{C}$ (DMSO-d $\left.{ }_{6}, \mathrm{ppm}\right): 14.8,39.3,89.6,108.2$, 112.3(3), 117.5, 120.9, 124.5(2), 124.9(2), 126.3(2), 129.0, 137.4(2), 142.8, 144.0, 155.6, 162.4, $187.6(\mathrm{C}=\mathrm{S})$. Anal. Calcd. for $\mathrm{C}_{22} \mathrm{H}_{16} \mathrm{~N}_{6} \mathrm{O}_{4} \mathrm{~S}$ (460): C, 57.38; H, 3.50; N, 18.25. Found: C, 57.69; H, 3.20; N, 18.56 .

3.3.4. 3-(4-Bromophenyl)-1-(1,5-dimethyl-3-oxo-2-phenyl-2,3-dihydro-1H-pyrazol-4-yl)-4-oxo-2thioxo-1,2,3,4-tetrahydropyrimidine-5-carbonitrile $\mathbf{1 3}$

Yield\%: 69, m.p. $=76.0{ }^{\circ} \mathrm{C}, \mathrm{IR}, \mathrm{cm}^{-1}: 3100$ (CH arom.), 2986, 2861 (CH aliph.), $2218(\mathrm{CN}), 1684$, $1653(2 \mathrm{C}=\mathrm{O}), 1216(\mathrm{C}=\mathrm{S}) .{ }^{1} \mathrm{H}\left(\mathrm{DMSO}-d_{6}, \mathrm{ppm}\right): 2.2\left[\mathrm{~s}, 3 \mathrm{H}, \mathrm{CH}_{3}\right], 3.1\left[\mathrm{~s}, 3 \mathrm{H}, \mathrm{N}-\mathrm{CH}_{3}\right], 7.0-7.6$ $[\mathrm{m}, 10 \mathrm{H}, \mathrm{Ar}-\mathrm{H}+\mathrm{CH}-$ pyrimidine $] .{ }^{13} \mathrm{C}$ (DMSO- $\left.d_{6}, \mathrm{ppm}\right): 13.9,39.5,92.4,104.6,113.8(2), 114.1$, 116.6, 119.9, 121.0(2), 127.2(2), 129.0, 132.6, 133.4(2), 137.7, 138.6, 159.9, 160.1, 186.6 (C=S). Anal. Calcd. for $\mathrm{C}_{22} \mathrm{H}_{16} \mathrm{BrN}_{5} \mathrm{O}_{2} \mathrm{~S}$ (493): C, 53.45; H, 3.26; N, 14.17. Found: C, 53.70; H, 3.11; N, 14.44 .

3.3.5. 1-(1,5-Dimethyl-3-oxo-2-phenyl-2,3-dihydro-1H-pyrazol-4-yl)-3-(4-iodophenyl)-4-oxo-2thioxo-1,2,3,4-tetrahydropyrimidine-5-carbonitrile $\mathbf{1 4}$

Yield\%: 90, m.p. $=114.1{ }^{\circ} \mathrm{C}, \mathrm{IR}, \mathrm{cm}^{-1}: 3092(\mathrm{CH}$ arom.), 2980, $2861(\mathrm{CH}$ aliph.), $2187(\mathrm{CN})$, 1684, $1654(2 \mathrm{C}=\mathrm{O}), 1276(\mathrm{C}=\mathrm{S}) .{ }^{1} \mathrm{H}\left(\mathrm{DMSO}-d_{6}, \mathrm{ppm}\right): 2.5\left[\mathrm{~s}, 3 \mathrm{H}, \mathrm{CH}_{3}\right], 3.1\left[\mathrm{~s}, 3 \mathrm{H}, \mathrm{N}-\mathrm{CH}_{3}\right]$, 7.6-7.9 [m, 10H, Ar-H + CH-pyrimidine]. ${ }^{13} \mathrm{C}$ (DMSO- $\left.d_{6}, \mathrm{ppm}\right): 13.9,39.5,88.8(2), 107.3,114.6(2)$, 116.2, 120.2, 123.6(2), 129.0(3), 130.6, 137.2, 137.9(2), 139.1, 162.7, 166.1, 187.3. Anal. Calcd. for $\mathrm{C}_{22} \mathrm{H}_{16} \mathrm{~N}_{5} \mathrm{O}_{2} \mathrm{~S}$ (541): C, 48.81; H, 2.98; N, 12.94. Found: C, 48.69; H, 2.69; N, 12.72.

3.3.6. 4-(5-Cyano-3-(1,5-dimethyl-3-oxo-2-phenyl-2,3-dihydro-1H-pyrazol-4-yl)-6-oxo-2-thioxo-2,3tetrahydropyrimidin-1 $(6 H)$-yl)benzensulfonamide 15

Yield\%: 90, m.p. $=164.8{ }^{\circ} \mathrm{C}, \mathrm{IR}, \mathrm{cm}^{-1}: 3041(\mathrm{CH}$ arom.), 2934, 2881 (CH aliph.), $2217(\mathrm{CN})$, 1714, $1662(2 \mathrm{C}=\mathrm{O}), 1296(\mathrm{C}=\mathrm{S}) .{ }^{1} \mathrm{H}\left(\mathrm{DMSO}-d_{6}, \mathrm{ppm}\right): 2.2\left[\mathrm{~s}, 3 \mathrm{H}, \mathrm{CH}_{3}\right], 3.0\left[\mathrm{~s}, 3 \mathrm{H}, \mathrm{N}-\mathrm{CH}_{3}\right]$, 7.3-8.0 [m, 11H, Ar-H $\left.+\mathrm{SO}_{2} \mathrm{NH}_{2}\right], 8.1[\mathrm{~s}, 1 \mathrm{H}, \mathrm{CH}-$ pyrimidine $] .{ }^{13} \mathrm{C}$ (DMSO-d $\left.d_{6}, \mathrm{ppm}\right): 13.9,39.4$, 96.2, 104.6, 114.2(2), 116.4, 117.2, 121.6(2), 125.1(2), 127.3(2), 129.1, 134.2, 134.5, 139.6, 140.9, 160.2, 160.5, $187.6(\mathrm{C}=\mathrm{S})$. Anal. Calcd. for $\mathrm{C}_{22} \mathrm{H}_{18} \mathrm{~N}_{6} \mathrm{O}_{4} \mathrm{~S}_{2}$ (494): C, 53.43; H, 3.67; N, 16.99 . Found: C, 53.69; H, 3.90; N, 16.34.

3.3.7. 3,3'-(4,4'-Sulfonylbis(4,1-phenylene))bis(1-(1,5-dimethyl-3-oxo-2-phenyl-2,3-dihydro-1Hpyrazol-4-yl)-4-oxo-2-thioxo-1,2,3,4-tetrahydropyrimidine-5-carbonitrile) 16

A mixture of 2 (0.01 mol) and 1-(4-isothiocyanatophenylsulfonyl)-4-isothiocyanatobenzene $(0.02 \mathrm{~mol})$ was refluxed for $10 \mathrm{~h}$ in ethanol containing sodium ethoxide $(0.01 \mathrm{~mol})$. The reaction mixture was cooled, poured onto ice water, acidified with dil. $\mathrm{HCl}$, the precipitated solid product was filtered and crystallized from methanol to give 16. Yield\%: 81, m.p. $=140.6{ }^{\circ} \mathrm{C}, \mathrm{IR}, \mathrm{cm}^{-1}: 3076$ (CH arom.), 2981, 2861 (CH aliph.), $2219(\mathrm{CN}), 1661,1643(4 \mathrm{C}=\mathrm{O}), 1322(2 \mathrm{C}=\mathrm{S}) .{ }^{1} \mathrm{H}$ (DMSO- $d_{6}$, ppm): 2.2 [s, 6H, 2CH 3$], 3.1\left[\mathrm{~s}, 6 \mathrm{H}, 2 \mathrm{~N}-\mathrm{CH}_{3}\right], 7.3-8.0$ [m, 18H, $\left.\mathrm{Ar}-\mathrm{H}\right], 8.6$ [s, 2H, 2CH-pyrimidine]. ${ }^{13} \mathrm{C}$ (DMSO- $\left.d_{6}, \mathrm{ppm}\right): 13.8(2), 39.0(2), 92.6(2), 108.6(2), 113.5(4), 114.1(2), 118.4(2), 121.7(4)$, 
127.0(4), 128.2(4), 129.1(2), 134.5(2), 136.2(2), 136.7(2), 142.4(2), 159.4(2), 160.2(2), 187.6(2). Anal. Calcd. for $\mathrm{C}_{44} \mathrm{H}_{32} \mathrm{~N}_{10} \mathrm{O}_{6} \mathrm{~S}_{3}$ (892): C, 59.18; H, 3.61; N, 15.69. Found: C, 59.40; H, 3.80; N, 15.48 .

\subsection{General Procedure for Preparation of Compounds $\mathbf{1 7}$ and $\mathbf{1 8}$}

Compound $10(0.01 \mathrm{~mol})$ was mixed with either hydrazine hydrate or phenyl hydrazine $(0.01 \mathrm{~mol})$ in dioxane $(20 \mathrm{~mL})$ and refluxed for $5 \mathrm{~h}$, the reaction mixture was cooled, poured onto ice water. The precipitated solid products were filtered and crystallized from methanol to give compounds $\mathbf{1 7}$ and $\mathbf{1 8}$, respectively.

3.4.1. (Z)-1-(1,5-Dimethyl-3-oxo-2-phenyl-2,3-dihydro-1H-pyrazol-4-yl)-2-hydrazono-4-oxo-3phenyl-1,2,3,4-tetrahydropyrimidine-5-carbonitrile $\mathbf{1 7}$

Yield\%: 68, m.p. $=78.0^{\circ} \mathrm{C}, \mathrm{IR}, \mathrm{cm}^{-1}: 3213,3191\left(\mathrm{NH}_{2}\right), 3047(\mathrm{CH}$ arom. $), 2961,2876(\mathrm{CH}$ aliph.), $2191(\mathrm{CN}) .{ }^{1} \mathrm{H}$ (DMSO-d, $\left.\mathrm{ppm}\right): 2.5\left[\mathrm{~s}, 3 \mathrm{H}, \mathrm{CH}_{3}\right], 3.2\left[\mathrm{~s}, 3 \mathrm{H}, \mathrm{N}-\mathrm{CH}_{3}\right], 4.5\left[\mathrm{~s}, 2 \mathrm{H}, \mathrm{NH}_{2}\right.$, $\mathrm{D}_{2} \mathrm{O}$-exchangeable], 7.1-7.5 [m, $11 \mathrm{H}, \mathrm{Ar}-\mathrm{H}+\mathrm{CH}-$ pyrimidine $] .{ }^{13} \mathrm{C}$ (DMSO- $\left.d_{6}, \mathrm{ppm}\right): 13.1,39.0$, 94.6, 106.2, 114.5(2), 116.7, 118.2, 120.6(2), 122.1, 127.6(2), 128.4(2), 129.7, 134.2, 137.7, 138.5, 156.2, 162.8, 168.9. Anal. Calcd. for $\mathrm{C}_{22} \mathrm{H}_{19} \mathrm{~N}_{7} \mathrm{O}_{2}$ (413): C, 63.91; H, 4.63; N, 23.72. Found: C, 63.70; $\mathrm{H}, 4.91 ; \mathrm{N}, 23.55$.

3.4.2. (Z)-1-(1,5-Dimethyl-3-oxo-2-phenyl-2,3-dihydro-1H-pyrazol-4-yl)-4-oxo-3-phenyl-2-(2phenylhydrazono)-1,2,3,4-tetrahydropyrimidine-5-carbonitrile 18

Yield\%: 65, m.p. $=90.6{ }^{\circ} \mathrm{C}, \mathrm{IR}, \mathrm{cm}^{-1}: 3381(\mathrm{NH}), 3074(\mathrm{CH}$ arom.), 2966, $2836(\mathrm{CH}$ aliph.), $2220(\mathrm{CN}), 1700,1653(2 \mathrm{C}=\mathrm{O}) .{ }^{1} \mathrm{H}\left(\mathrm{DMSO}-d_{6}, \mathrm{ppm}\right): 2.4\left[\mathrm{~s}, 3 \mathrm{H}, \mathrm{CH}_{3}\right], 3.2\left[\mathrm{~s}, 3 \mathrm{H}, \mathrm{N}-\mathrm{CH}_{3}\right], 7.0-8.0$ $[\mathrm{m}, 15 \mathrm{H}, \mathrm{Ar}-\mathrm{H}], 8.4\left[\mathrm{~s}, 1 \mathrm{H}, \mathrm{CH}-\right.$ pyrimidine], $9.5\left[\mathrm{~s}, 1 \mathrm{H}, \mathrm{NH}, \mathrm{D}_{2} \mathrm{O}\right.$-exchangeable]. ${ }^{13} \mathrm{C}$ (DMSO- $d_{6}$, ppm): 14.1, 39.6, 95.3, 108.3, 113.8(2), 114.6, 115.8, 116.4(2), 118.9, 119.5(2), 122.2, 128.6(2), 129.0(2), 129.6, 129.8(2), 130.2, 139.2, 141.5, 144.6, 153.5, 155.7, 165.6. Anal. Calcd. for $\mathrm{C}_{28} \mathrm{H}_{23} \mathrm{~N}_{7} \mathrm{O}_{2}$ (489): C, 68.70; H, 4.74; N, 20.03. Found: C, 68.92; H, 4.99; N, 20.33.

3.4.3. (Z)-4-(3-Amino-6-hydrazono-7-phenyl-6,7-dihydropyrazolo[3,4- $d$ ]pyrimidin-5-yl)-1,5dimethyl-2-phenyl-1,2-dihydropyrazol-3-one 19

Compound $10(0.01 \mathrm{~mol})$ was mixed with hydrazine hydrate $(0.02 \mathrm{~mol})$ in dioxane $(20 \mathrm{~mL})$ and refluxed for $5 \mathrm{~h}$, the reaction mixture was cooled, poured onto ice water. The precipitated solid products were filtered and crystallized from methanol to give compounds 19. Yield\%: 73, m.p. $=222.6{ }^{\circ} \mathrm{C}$, IR, cm ${ }^{-1}$ : 3420, $3362\left(\mathrm{NH}_{2}\right), 3050$ (CH arom.), 2971, $2861(\mathrm{CH}$ aliph.), $1654(\mathrm{C}=\mathrm{O}), 1617(\mathrm{C}=\mathrm{N})$. ${ }^{1} \mathrm{H}$ (DMSO- $\left.d_{6}, \mathrm{ppm}\right): 2.2\left[\mathrm{~s}, 3 \mathrm{H}, \mathrm{CH}_{3}\right], 3.2\left[\mathrm{~s}, 3 \mathrm{H}, \mathrm{N}-\mathrm{CH}_{3}\right], 5.4$ [s, 2H, N- $\mathrm{NH}_{2}, \mathrm{D}_{2} \mathrm{O}$-exchangeable], $6.5\left[\mathrm{~s}, 2 \mathrm{H}, \mathrm{NH}_{2}, \mathrm{D}_{2} \mathrm{O}\right.$-exchangeable], 7.0-8.1 [m, 11H, Ar-H $+\mathrm{CH}-$ pyrimidine]. ${ }^{13} \mathrm{C}$ (DMSO- $d_{6}$, ppm): 11.4, 39.0, 97.6, 107.3, 112.6(2), 114.3(2), 116.7, 118.6, 123.7, 128.6(2), 129.1, 129.4(2), 134.1, 139.8, 155.7, 162.3, 166.0(2). Anal. Calcd. for $\mathrm{C}_{22} \mathrm{H}_{21} \mathrm{~N}_{9} \mathrm{O}$ (427): C, 61.81; H, 4.95; N, 29.49 . Found: C, 62.03; H, 5.12; N, 29.67. 


\subsection{General Procedure for Preparation of Compounds $\mathbf{2 0}$ and $\mathbf{2 1}$}

A solution of 4-aminoantipyrine $1(0.001 \mathrm{~mol})$ in either triethylorthoformate or triethylorthoacetate $(0.001 \mathrm{~mol})$ containing three drops of acetic anhydride was refluxed for $8 \mathrm{~h}$, the reaction mixture was cooled and then poured onto cold water, the obtained solid was recrystallized from methanol to give compounds $\mathbf{2 0}$ and $\mathbf{2 1}$, respectively.

\subsection{1. (E)-Ethyl- $N$-1,5-dimethyl-3-oxo-2-phenyl-2,3-dihydro-1H-pyrazol-4-yl-formimidate 20}

Yield\%: 90, m.p. $=196.9{ }^{\circ} \mathrm{C}, \mathrm{IR}, \mathrm{cm}^{-1}: 3078$ (CH arom.), 2976, $2912(\mathrm{CH}$ aliph.), $1661(\mathrm{C}=\mathrm{O})$, $1612(\mathrm{C}=\mathrm{N}) .{ }^{1} \mathrm{H}\left(\mathrm{DMSO}-d_{6}, \mathrm{ppm}\right): 1.0\left[\mathrm{t}, 3 \mathrm{H}, \mathrm{CH}_{3}\right], 2.3\left[\mathrm{~s}, 3 \mathrm{H}, \mathrm{CH}_{3}\right], 3.1\left[\mathrm{~s}, 3 \mathrm{H}, \mathrm{N}-\mathrm{CH}_{3}\right], 3.5[\mathrm{q}, 2 \mathrm{H}$, $\mathrm{CH}_{2}$ ], 7.2-7.5 [m, 5H, Ar-H], $8.1[\mathrm{~s}, 1 \mathrm{H}, \mathrm{CH}] .{ }^{13} \mathrm{C}$ (DMSO- $\left.d_{6}, \mathrm{ppm}\right): 11.3,18.5,39.3,56.0,106.3$, 107.0(2), 120.0, 129.0(2), 135.1, 151.6, 160.1, 164.7. Anal. Calcd. for $\mathrm{C}_{14} \mathrm{H}_{17} \mathrm{~N}_{3} \mathrm{O}_{2}$ (259): C, 64.85; H, 6.61; N, 16.34. Found: C, 64.66; H, 6.34; N, 16.67.

\subsection{2. (E)-Ethyl- $N$-1,5-dimethyl-3-oxo-2-phenyl-2,3-dihydro-1H-pyrazol-4-yl-acetimidate 21}

Yield\%: 88, m.p. $=81.6^{\circ} \mathrm{C}, \mathrm{IR}, \mathrm{cm}^{-1}: 3086$ (CH arom.), 2936, 2817 (CH aliph.), $1658(\mathrm{C}=\mathrm{O}), 1598$ $(\mathrm{C}=\mathrm{N}) .{ }^{1} \mathrm{H}\left(\mathrm{DMSO}-d_{6}, \mathrm{ppm}\right): 1.2\left[\mathrm{t}, 3 \mathrm{H}, \mathrm{CH}_{3}\right], 1.9\left[\mathrm{~s}, 3 \mathrm{H}, \mathrm{N}=\mathrm{C}-\mathrm{CH}_{3}\right], 2.1\left[\mathrm{~s}, 3 \mathrm{H}, \mathrm{CH}_{3}\right], 3.3[\mathrm{~s}, 3 \mathrm{H}$, $\left.\mathrm{N}-\mathrm{CH}_{3}\right], 4.1\left[\mathrm{q}, 2 \mathrm{H}, \mathrm{CH}_{2}\right], 7.2-7.5[\mathrm{~m}, 5 \mathrm{H}, \mathrm{Ar}-\mathrm{H}] .{ }^{13} \mathrm{C}$ (DMSO-d, $\left.\mathrm{ppm}\right): 10.1\left(\mathrm{CH}_{3}-\right.$ pyrazole), 14.0 $\left(\mathrm{CH}_{3}\right.$-ethyl), $17.4\left(\mathrm{~N}=\mathrm{C}-\mathrm{CH}_{3}\right), 39.1\left(\mathrm{~N}-\mathrm{CH}_{3}\right), 61.0\left(\mathrm{CH}_{2}\right.$-ethyl), 112.6, 118.7(2), 122.8, 128.9(2), 135.4, 146.1, 160.0, 164.0 (C=O). Anal. Calcd. for $\mathrm{C}_{15} \mathrm{H}_{19} \mathrm{~N}_{3} \mathrm{O}_{2}$ (273): C, 65.91; H, 7.01; N, 15.37 . Found: C, 66.13; H, 7.34; N, 15.48 .

\subsection{In-Vitro Anticancer Screening}

The human tumor cell line (MCF7) was available from the National Cancer Institute, Cairo, Egypt. The antitumor activity of the newly synthesized compounds against the MCF7 cells was measured using the Sulforhodamine B (SRB) assay by the method of Skehan et al. (1990) [23,24]. The cell lines were grown in RPMI 1640 medium containing 10\% fetal bovine serum and $2 \mathrm{mM}$ L-glutamine. Cells were plated in 96-multi-well plates $\left(10^{4}\right.$ cells/well) and were incubated at $37{ }^{\circ} \mathrm{C}, 5 \% \mathrm{CO}_{2}$ in a humidified atmosphere for $24 \mathrm{~h}$ to allow attachment prior to addition of compounds. The test compounds 3-14 were dissolved in DMSO and diluted with saline to the appropriate volume and maintained in RPMI 1640 medium. Different concentrations of the compounds under test were made: 5, 12.5, 25, 30 and $50 \mu \mathrm{M}$ and were added to the cells. Triplicate wells were prepared for each individual dose. Cells were incubated with the compounds for $48 \mathrm{~h}$ at $37{ }^{\circ} \mathrm{C}, 5 \% \mathrm{CO}_{2}$. After $48 \mathrm{~h}$, cells were fixed in situ by the gentle addition of $50 \mu \mathrm{L}$ of cold $30 \%(w / v)$ trichloroacetic acid (TCA) (final concentration, $10 \%$ ) and incubated for $60 \mathrm{~min}$ at $4{ }^{\circ} \mathrm{C}$. The supernatant was discarded and the plates were washed five times with tap water and air dried. Sulforhodamine B (SRB) solution $(50 \mu \mathrm{L})$ at $0.4 \%(w / v)$ in $1 \%$ acetic acid was added to each well and plates were incubated for 20 min at room temperature. After staining, unbounded dye was removed by four washes with $1 \%$ acetic acid, and attached stain was recovered with Tris-EDTA buffer. Color intensity was measured at wave length $564 \mathrm{~nm}$ in an ELISA reader (Gmbh, Viesbaden, Germany). The relation between surviving fraction and drug concentration was plotted to get the survival curve from each compound after the 
specified time. The concentration required for $50 \%$ inhibition of cell viability $\left(I C_{50}\right)$ was calculated and compared with the reference drug doxorubicin and the results are given in Table 1.

\section{Conclusions}

The objective of the present study was to synthesize and investigate the anticancer activity of some novel pyrazole carrying a biologically active sulfonamide moieties. It was found that compounds 5, 13, 14, and 16-19 showed promising anticancer activity, higher than that of doxorubicin as reference drug against human breast cancer cell line (MCF7), while compounds 10, 12 are nearly as active as doxorubicin as positive control. Compound 8 exhibited a moderate activity and compounds 2-4, 6, 7, 9, and 20 showed a weak activity, while compounds 11, 15 and 21 revealed no activity. Further investigations on different probable mechanisms of action and dose-response studies should be helpful in identifying the specific site(s) of action and optimum doses of the synthesized antipyrine derivatives. These investigations would be crucial in discovering more potent and more selective anti-breast cancer agents.

\section{Acknowledgments}

The authors would like to extend their sincere appreciation to the Deanship of Scientific Research at King Saud University (Riyadh, Saudi Arabia) for its funding of this research through the Research Group Project No. RGP-VPP-302.

\section{Author Contributions}

M.M.G. suggested the research idea, contributed in the experimental work and in writing the paper. M.G.E.-G. contributed in experimental work and in writing the paper. M.S.A. contributed in the experimental work, biological activity and in writing the paper.

\section{Conflicts of Interest}

The authors declare no conflict of interest.

\section{References}

1. Himly, M.; Jahn-Schmid, B.; Pittertschatscher, K.; Bohle, B.; Grubmayr, K.; Ferreira, F.; Ebner, H.; Ebner, C. Ig E-mediated immediate-type hypersensitivity to the pyrazolone drug propyphenazone. J. Allergy Clin. Immunol. 2003, 111, 882-888.

2. Gürsoy, A.; Demirayak, S.; Capan, G.; Erol, K.; Vural, K. Synthesis and preliminary evaluation of new 5-pyrazolinone derivatives as analgesic agents. Eur. J. Med. Chem. 2000, 35, 359-364.

3. Teng, Y.; Liu, R.; Li, C.; Zhang, H. Effect of 4-aminoantipyrine on oxidative stress induced by glutathione depletion in single human erythrocytes using a microfluidic device together with fluorescence imaging. J. Hazard. Mater. 2011, 192, 1766-1771.

4. Turan-Zitouni, G.; Sivaci, M.; Kiliç, F.S.; Erol, K. Synthesis of some triazolyl-antipyrine derivatives and investigation of analgesic activity. Eur. J. Med. Chem. 2001, 36, 685-689. 
5. Lutsevich, A.N.; Bender, K.I.; Reshet'ko, O.V. The relationship between antipyrine kinetics, the seromucoid content and the xanthine oxidase activity in the plasma of rats with acute and chronic inflammation. Eksp Klin. Farmakol. 1995, 58, 51-55.

6. Bondock, S.; Rabie, R.; Etman, H.A.; Fadda, A.A. Synthesis and antimicrobial activity of some new heterocycles incorporating antipyrine moiety. Eur. J. Med. Chem. 2008, 43, 2122-2129.

7. Metwally, M.A.; Gouda, M.A.; Harmal, A.N.; Khalil, A.M. Synthesis, antitumor, cytotoxic and antioxidant evaluation of some new pyrazolotriazines attached to antipyrine moiety. Eur. J. Med. Chem. 2012, 56, 254-262.

8. Kakiuchi, Y.; Sasaki, N.; Satoh-Masuoka, M.; Murofushi, H.; Murakami-Murofushi, K. A novel pyrazolone, 4,4-dichloro-1-(2,4-dichlorophenyl)-3-methyl-5-pyrazolone, as a potent catalytic inhibitor of human telomerase q. Biochem. Biophy. Res. Commun. 2004, 320, 1351-1358.

9. Sigroha, S.; Narasimhan, B.; Kumar, P.; Khatkar, A.; Ramasamy, K.; Mani, V.; Mishra, R.K.; Abdul Majeed, A.B. Design, synthesis, antimicrobial, anticancer evaluation, and QSAR studies of 4-(substituted benzylidene-amino)-1,5-dimethyl-2-phenyl-1,2-dihydropyrazol-3-ones. Med. Chem. Res. 2012, 21, 3863-3875.

10. Chandrasekharan, N.V.; Dai, H.; Roos, K.L.T.; Evanson, N.K.; Tomsik, J.; Elton, T.S. COX-3, a cyclooxygenase-1 variant inhibited by acetaminophen and other analgesic/antipyretic drugs: Cloning, structure, and expression. Proc. Natl. Acad. Sci. USA 2002, 99, 13926-13931.

11. Levy, M. Hypersensitivity to pyrazolones. Thorax 2000, 55, 72-74.

12. Al-Haiza, M.A.; El-Assiery, S.A.; Sayed, G.H. Synthesis and potential antimicrobial activity of some new compounds containing the pyrazol-3-one moiety. Acta Pharm. 2001, 51, 251-261.

13. Lin, R.; Chiu, G.; Yu, Y. Design, synthesis, and evaluation of 3,4-disubstituted pyrazole analogues as anti-tumor CDK inhibitors. Bioorg. Med. Chem. Lett. 2007, 17, 4557-4581.

14. Aly, H.M.; El-Gazzar, M.G. Novel pyrazole derivatives as anticancer and radiosensitizing agents. Arzneimittelforschung 2012, 62, 105-112.

15. Ghorab, M.M.; Ragab, F.A.; Heiba, H.I.; Hanan, A.Y.; El-Gazzar, M.G. Synthesis of novel pyrazole and pyrimidine derivatives bearing sulfonamide moiety as antitumor and radiosensitizing agents. Med. Chem. Res. 2012, 21, 1376-1383.

16. Ghorab, M.M.;Heiba, H.I.; Hassan, A.A.; Abd El-Aziz, A.B.; El-Gazzar, M.G. Antimicrobial evaluation of novel pyrrole, pyrazole, pyrimidine and pyrrolo [2,3-d]-pyrimidine derivatives brearing sulfonamide moiety. J. Am. Sci. 2011, 7, 1064-1073.

17. Ghorab, M.M.; Ragab, F.A.; Heiba, H.I.; Youssef, H.A.; Galal, M. Synthesis of some new pyrazole and pyrimidine derivatives carrying a sulfonamide moiety of expected antitumor activity and study of the synergistic effect of gamma-irradiation. Arzneimittelforschung 2010, 60, 48-55.

18. Ghorab, M.M.; Alsaid, M.S.; Nissan, Y.M. Dapson in heterocyclic chemistry, part V: Synthesis, molecular docking and anticancer activity of some novel sulfonylbiscompounds carrying biologically active dihydropyridine, dihydroisoquinoline, 1,3-dithiolan, 1,3-dithian, acrylamide, pyrazole, pyrazolopyrimidine and benzochromenemoieties. Chem. Pharm. Bull. (Tokyo) 2012, 60, 1019-1028.

19. Mohareb, R.M.; Sherif, S.M.; Zohdi, H.F. Heterocyclic synthesis with enamines: Convenient synthesis of polyfunctionally substituted pyrazole, pyridine, pyrimidine and pyrazolo[3,4-d]pyrimidine derivatives. J. Chin. Chem. Soc. 1993, 40, 181-187. 
20. Mohamed, A.M.; El-Sayed, W.A.; Alsharari, M.A.; Al-Qalawi, H.R.; Germoush, M.O. Anticancer activities of some newly synthesized pyrazole and pyrimidine derivatives. Arch. Pharm. Res. 2013, 36, 1055-1065.

21. Ghorab, M.M.; Ragab, F.A.; Heiba, H.I.; Youssef, H.A.; El-Gazzar, M.G. Synthesis of novel pyrrole and pyrrolo[2,3- $d$ ]pyrimidine derivatives bearing sulfonamide moiety for evaluation as anticancer and radiosensitizing agents. Bioorg. Med. Chem. Lett. 2010, 20, 6316-6320.

22. Al-Issa, S.A. Synthesis and anticancer activity of some fused pyrimidines and related heterocycles. Saudi Pharm. J. 2013, 21, 305-316.

23. Skehan, P.; Storeng, R.; Scudiero, D.; Monks, A.; McMahon, J.; Vistica, D.; Warren, J.T.; Bokesch, H.; Kenney, S.; Boyd, M.R. New colorimetric cytotoxicity assay for anticancer-drug screening. J. Natl. Cancer Inst. 1990, 82, 1107-1112.

24. Rubinstein, L.V.; Shoemaker, R.H.; Paull, K.D.; Simon, R.M.; Tosini, S.; Skehan, P.; Scudiero, D.A.; Monks, A.; Boyd, M.R. Comparison of in vitro anticancer-drug-screening data generated with a tetrazolium assay $v s$. a protein assay against a diverse panel of human tumor cell lines. J. Natl. Cancer Inst. 1990, 82, 1113-1118.

(C) 2014 by the authors; licensee MDPI, Basel, Switzerland. This article is an open access article distributed under the terms and conditions of the Creative Commons Attribution license (http://creativecommons.org/licenses/by/3.0/). 